\title{
EVALUACIÓN FÍSICO-QUÍMICA Y MICROBIOLÓGICA DEL SISTEMA DE AGUA QUE ABASTECE A LAS PLANTAS PROCESADORAS DE QUESO FRESCO ARTESANAL DE LA PARROQUIA QUIMIAG- RIOBAMBA-ECUADOR
}

\author{
PHYSICO -CHEMICAL AND MICROBIOLOGICAL ASSESSMENT OF WATER \\ SUPPLY SYSTEM, THAT PROVIDES WATER TO HANDMADE FRESH CHEESE \\ FACTORIES LOCATED IN QUIMIAG-RIOBAMBA-ECUADOR
}

\author{
${ }^{1}$ Paola Arguello-Hernández ${ }^{*},{ }^{1}$ Ligia Carrillo, ${ }^{1,2}$ Sandra Escobar-Arrieta, ${ }^{2}$ Nelly Guananga-Diaz, \\ ${ }^{3}$ Félix Andueza, ${ }^{1,2}$ Ana Albuja-Landi
}

${ }^{1}$ Seguridad Alimentaria Grupo de Investigación y Desarrollo “SAGID”, Escuela Superior Politécnica de Chimborazo, Facultad de Ciencias, Riobamba, Ecuador

${ }^{2}$ Leishmaniosis y otras Parasitosis en el Ecuador "LEISHPAREC”, Escuela Superior Politécnica de Chimborazo, Facultad de Ciencias, Riobamba, Ecuador

${ }^{3}$ Universidad Central del Ecuador, Quito, Ecuador

*p_farguello@espoch.edu.ec

La calidad del agua utilizada en las plantas de procesamiento de alimentos, influye en la calidad del producto final, siendo necesario su análisis y control. Este estudio analizó los parámetros físicoquímicos y microbiológicos del sistema de agua que abastece a las queseras artesanales, ubicadas en la parroquia rural Quimiag. Las muestras fueron tomadas por triplicado en época de verano en 14 puntos del sistema de abastecimiento de agua. Estos puntos fueron las vertientes de agua natural, los tanques de almacenamiento y los grifos en las queseras y sus tanques de reserva. Los análisis efectuados fueron: turbiedad, color, $\mathrm{pH}$, conductividad, sólidos totales, nitratos, nitritos, fluoruros, coliformes fecales, y parásitos, según las normas técnicas ecuatorianas y una norma mexicana. Los resultados evidencian que el agua proveniente de todos los puntos de muestreo cumple con los parámetros físico-químicos que exige la norma NTE INEN 1108 (requisitos agua potable), sin embargo, sólo las fuentes naturales de agua (vertientes) presentan calidad microbiológica aceptable, y a medida que el agua continúa en el sistema de abastecimiento, ésta presenta mayor carga bacteriana y presencia de parásitos. Al no contar con una planta de tratamiento que garantice la calidad del agua utilizada en las queseras, ésta debe ser clorada adecuadamente para eliminar los microorganismos y prevenir la contaminación cruzada.

Palabras claves: queseras artesanales, calidad de agua, coliformes fecales, parásitos.

The quality of the water used in food processing plants, affects the quality of the final product, therefore its analysis and control are needed. This study analyzed the physico-chemical and microbiological parameters of the water supply system that provides to the handmade cheese factories located in Quimiag, a rural area in Ecuador. Fourteen points were sampled along the system water supply ( 3 natural water sources, 3 catchment tanks, 1 storage and deliverated tank, 5 faucets and 2 storage tanks located in the handmade cheese factories). At each point triplicate samples were taken. The analyses carried out were: turbidity, color, $\mathrm{pH}$, conductivity, total solids, nitrates, nitrites, fluorides, fecal coliform bacteria, and parasites. These were did according to Ecuadorian and Mexican technical standars. The results show that water from all sampling points meets the physico- chemical parameters required by the standard NTE INEN 1108 (Ecuadorian drinking water requirements), but only the samples from the natural water sources meet to microbiological parameters, but it keeps increasing the bacteria and parasites counting when the water flows along the system. As long as Quimiag does not have a water treatment plant, the 
handmade cheese factories and the population in general should chlorinate the water in order to kill microorganisms and avoid cross-contamination.

Keywords: handmade cheese factories, water quality, fecal coliform bacteria, parasites.

Fecha de recepción: 11-07-2018

Fecha de aceptación: 23-01-2019

\section{INTRODUCCIÓN}

La inocuidad de los alimentos es un aspecto fundamental de salud pública $y$ uno de los temas de mayor atención para los gobiernos, productores y consumidores (1).

Estudios realizados indican la relación de contaminación microbiana de los alimentos con las condiciones higiénicas de las instalaciones de fabricación (2), siendo necesaria la evaluación de todos los factores más influyentes sobre la calidad microbiológica del producto final. En una quesera, los principales puntos críticos de control son: la leche (materia prima), salmuera (adición de sal a los quesos una vez elaborados), ambiente, superficies vivas e inertes, medidas de higiene y el agua (3).

En Ecuador, las queseras artesanales generalmente están ubicadas en las zonas rurales; en la parroquia de Quimiag del cantón Riobamba, provincia de Chimborazo, existen 12 queseras identificadas por el Gobierno Autónomo Descentralizado Parroquial y el Ministerio de Industria y Productividad (MIPRO). El sistema de agua que abastece a Quimiag no es potable, el líquido proviene de dos vertientes, este se almacena en tanques y posteriormente se distribuye a los usuarios sin un control de calidad que garantice su seguridad.

La calidad del agua de abastecimiento es un factor importante que puede afectar la seguridad de los alimentos procesados; en esta industria, el agua es utilizada en el proceso de limpieza y desinfección de áreas en contacto con el alimento, $y$ como ingrediente en la preparación de salmuera. El uso de agua que no ha recibido un tratamiento que garantice las características de agua potable, sumado a las condiciones de manipulación en las queseras artesanales, la convierten en un factor de contaminación del queso fresco $(3,4)$ un producto que por su alta actividad de agua y su valor nutricional es catalogado como un alimento de alto riesgo microbiológico (5).

En un estudio previo (6) en el que fue evaluada la calidad microbiológica de la materia prima, suero y producto terminado en las queseras artesanales ubicadas en la parroquia rural Quimiag, indican que a pesar de que la leche tratada térmicamente que se utiliza para la elaboración cumple con la normativa el producto final presentó deficiente calidad microbiológica, existiendo lo que sugiere que existe contaminación en las siguientes etapas de elaboración y considerando que la industria de alimentos usa cantidades substanciales de agua en todos sus procesos, particularmente la industria dela leche, esta se puede convertir en un vector de contaminación del alimento (7).

Por lo anterior el propósito de este estudio fue evaluar la calidad físico-química y microbiológica del sistema de agua que abastece a las queseras del sector, proporcionando información relevante sobre la relación entre manipulación del agua (desde su fuente natural hasta su uso en la planta de producción) y la calidad del producto final.

\section{MATERIALES Y MÉTODOS}

Este estudio analizó la calidad física-química y microbiológica del sistema de agua que abastece a la parroquia Quimiag. La población se aprovisiona de dos fuentes naturales las cuales se encuentran ubicadas en las quebradas: Paquisha, Balcashi y Guntus.(Imagen 1)

Esto se realizó mediante análisis in situ y en el laboratorio, las muestras de agua fueron tomadas en 14 puntos del sistema de abastecimiento de agua. Tres provinieron de las vertientes (VA, VB y VC), tres de los tanques de captación asociados cada uno a una vertiente (TCA, TCB, TCC), otra muestra correspondió al tanque general de 
almacenamiento y distribución que recolecta el agua de los tres tanques de captación (TG), cinco muestras de los grifos de cinco queseras(Q1-Q5) y dos de tanques reservorios existentes en dos queseras (TRQ4 y TRQ5).

Se realizó el muestreo considerando la norma NTE INEN 2169:2013 (Agua. Calidad del agua. Muestreo. Manejo y conservación de muestras) (8) y la norma NTE INEN 1105 (muestreo para el análisis microbiológico) (9). Durante el muestreo in situ se determinó $\mathrm{pH}$, temperatura, conductividad y sólidos totales con el multiparámetro CONSORT C562@. Las cuantificaciones de color, turbidez, flúor, nitritos y nitratos se realizaron por el método espectrofotométrico con el uso del equipo HAHC $\mathrm{DR} / 2800 \AA$.

Los procedimientos se describen detalladamente en los manuales de cada equipo. El análisis de coliformes fecales se ejecutó con la técnica de fermentación de tubos múltiples, usando pruebas presuntivas y confirmativas para la cuantificación del número más probable (NMP), según lo descrito en la norma Mexicana NMXAA-042-SCFI-2015 (10). El análisis de los parásitos (Ameba coli, Giardia lamblai, Cryptosporidium sp. y otros parástios) se realizó por el método de centrifugación y flotación de Willis, para la determinación de Cryptosporidium sp. el producto obtenido se observó al microscopio óptico tras tinción de Ziehl-Neelsen $(11,12)$.

Todos los resultados fueron comparados con los requisitos establecido en la norma de requisitos de agua potable NTE INEN 1108:2014 (13).

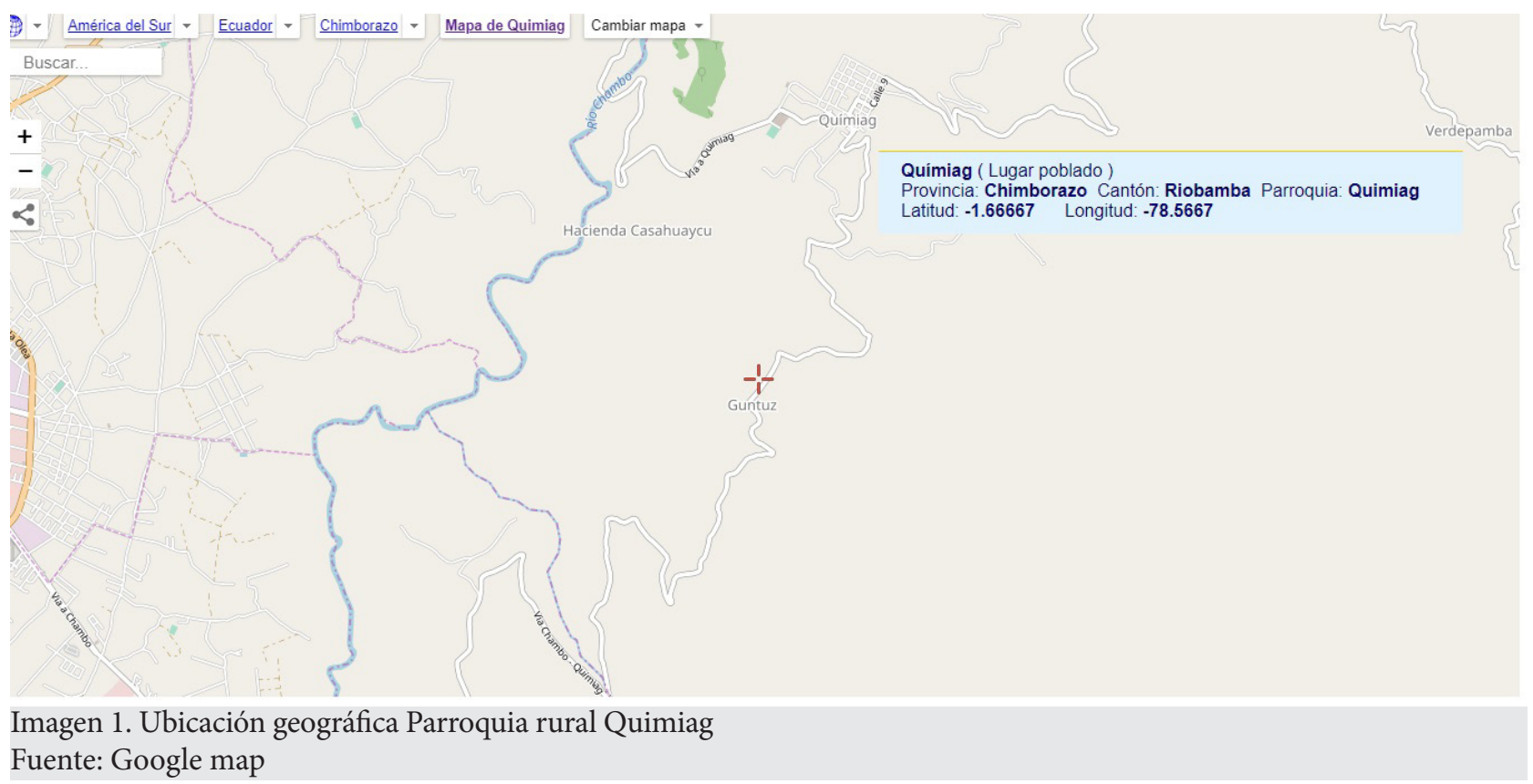

\section{RESULTADOS Y DISCUSIÓN}

La calidad microbiológica del agua de consumo en la parroquia Quimiag se evaluó en términos del NMP de coliformes fecales y ausencia o presencia de parásitos. En la tabla 1 se observan los resultados de los 14 puntos de muestreo. Se evidencia que únicamente en las vertientes (VA, VB y VC) no hubo presencia de microorganismos, por tanto, cumplen con los requisitos de la norma técnica ecuatoriana INEN 1108:2014. (Agua Potable. Requisitos); cuyos límites permitidos se muestran en las Tablas 1 y 2 . Comparando la normativa nacional con lo establecido en el Real Decreto 140/2013 del 7 de febrero en el que se indican los criterios sanitarios de la Calidad de Agua de consumo humano en España, los límites permitidos para los parámetros evaluados son similares a excepción del valor para nitritos, en España corresponde a 0,5 mg/L y $0.1 \mathrm{mg} / \mathrm{L}$ en la red de distribución y en el tanque de depósito respectivamente, y únicamente analizan parásitos cuando hay presencia de Clostridium perfringens (incluidas las esporas). 
A pesar de que existen datos de estudios realizados en vertientes y manantiales que indican que esta fuentes están contaminadas con coliformes fecales debido al pastoreo, maleza y cruce con aguas residuales (14), en las vertientes analizadas en Quimiag no se detectó estos microorganimos. Siendo estas vertientes el inicio del sistema de distribución del agua en la comunidad son las únicas muestras que cumplen con el parámetro microbiológico por tratarse de un tipo de fuente natural que se ubica en una zona montañosa, donde no existe presencia de animales de sangre caliente ni poblaciones aledañas por su acceso limitado, esto se pudo evidenciar durante el muestreo.

Considerando que el grupo de bacterias denominado coliformes fecales, están siempre presentes en la flora intestinal de animales de sangre caliente (14) y aunque algunas especies están ampliamente distribuidas en la naturaleza, su presencia indica tratamiento inadecuado del agua o su contaminación posterior (15), esto concuerda con los resultados de los siguientes puntos de muestreo a medida que el fluido avanza por las tuberías de PVC hasta el tanque de almacenamiento y distribución (TG), se observa aumento de coliformes fecales.

En las muestras de los grifos y los tanques reservorios de las dos queseras, los resultados son inferiores al TG, esto puede deberse a que no fueron tomadas las muestras en el mismo día, siendo las condiciones climáticas diferentes, concordando con la investigación (17) donde analizaron la Presencia de indicadores microbiológicos en agua para consumo humano en la localidad de San Cosme, Provincia de Corrientes (Argentina), los resultados de NMP de coliformes fecales varió según los distintos puntos de muestreo y entre las diferentes fechas de toma de muestra del agua de pozo. Los puntos de muestreo consecutivos a las vertientes pudieron haberse contamina- do a causa de los hábitos de defecación a campo abierto y a la presencia de animales domésticos y silvestres en las zonas rurales que actúan como reservorios de agentes patógenos, y contaminarse por fugas en las tuberías, además de que los tanques no tiene barreras que eviten contacto con el medio externo (17).

En referencia a los resultados hallados en las muestras de los grifos de las queseras, según estudios sobre la calidad del agua en la industria alimentaria, aun cuando el agua potable empleada tiene calidad sanitaria aceptable, el producto final puede no cumplir con la normativa correspondiente tras la contaminación del líquido al ingreso del sistema de distribución (18).

En este contexto, la calidad de la leche cruda y sus derivados puede verse comprometida por la calidad del agua de suministro que se utiliza para todas las actividades en la comunidad (19) ya que no cumple con los requisitos de la norma para agua potable (NTE INEN 1108 Requisitos Agua Potable) (11); es importante indicar que los tanques reservorios podrían considerarse foco de contaminación si este no tiene el manejo adecuado.

La norma pertinente en requisitos microbiológicos indica también la evaluación de Cryptosporidium y de Giardia lamblia, estos parásitos no deben estar presentes en agua potable, adicional se determinó la presencia de Ameba coli y de otros parásitos que se describen en la Tabla 1 . Cryptosporidium, un parásito que esta globalmente distribuido y un número de especies son reconocidas como patógenos humanos (20), los resultados muestran ausencia en todas las muestras analizas, no obstante, existió la presencia de otros parásitos en todas las muestras a excepción de las provenientes de las vertientes. En el $82 \%$ de muestras hay presencia de Giardia lamblia, $65 \%$ de Ambea coli y 59 \% de otros parásitos. Giardia lamblia, es el que más influye en la salud de los consumidores, especialmente en la población infantil, ya que se adosa en las microvellosidades del intestino delgado y evita la absorción de los alimentos, provocando desnutrición (21).

Estos parásitos ingresan por vía oral, por tanto, existe la probabilidad que se encuentre en el producto elaborado por las queseras artesanales, ya que el agua en estas condiciones es la que utilizan para todos los procesos en la planta procesamiento, teniendo consecuencias negativas no solamente para los consumidores de la comunidad sino también para los consumidores de los productos elaborados en las queseras. 


\begin{tabular}{|c|c|c|c|c|c|}
\hline $\begin{array}{l}\text { MUESTRA/ } \\
\text { MICROOR- } \\
\text { GANIMOS }\end{array}$ & $\begin{array}{c}\text { Coliformes } \\
{ }^{*} \text { Fecales (NM- } \\
\text { P/100mL) }\end{array}$ & $\begin{array}{c}\text { Ameba Coli } \\
\text { (Muestra } \\
\text { 5L) }\end{array}$ & $\begin{array}{c}{ }^{*} \text { Cryptosporidium } \\
\text { (Muestra 2L) }\end{array}$ & $\begin{array}{c}{ }^{* *} \text { Giardia } \\
\text { lamblia } \\
\text { (Muestra 5L) }\end{array}$ & $\begin{array}{c}\text { OTROS } \\
\text { PARÁSITOS } \\
\text { (Muestra 4L) } \\
\end{array}$ \\
\hline VA & 1,1 & Ausencia & Ausencia & Ausencia & Ausencia \\
\hline TCA & 2,6 & Ausencia & Ausencia & Presencia & $\begin{array}{l}\text { Hifas de Hongos, Tri- } \\
\text { churis parvum }\end{array}$ \\
\hline VB & 1,1 & Ausencia & Ausencia & Ausencia & Ausencia \\
\hline TCB & 2,6 & Presencia & Ausencia & Presencia & $\begin{array}{l}\text { Hifas de hongos, Para- } \\
\text { mecio }\end{array}$ \\
\hline VC & 1,1 & Presencia & Ausencia & Ausencia & $\begin{array}{l}\text { Entoameba histolytica, } \\
\text { hongos, }\end{array}$ \\
\hline TCC & 2,6 & Presencia & Ausencia & Presencia & Hongos, paramecio \\
\hline TG & 8,0 & Presencia & Ausencia & Presencia & $\begin{array}{l}\text { Entoameba histolytica, } \\
\text { Paramecio, hongos, } \\
\text { Trichuris trichura }\end{array}$ \\
\hline GQ1 & 4,6 & Presencia & Ausencia & Presencia & Paramecio \\
\hline GQ2 & 4,6 & Presencia & Ausencia & Presencia & $\begin{array}{l}\text { Entoameba histolytica, } \\
\text { Paramecio }\end{array}$ \\
\hline GQ3 & 2,6 & Ausencia & Ausencia & Presencia & Paramecio \\
\hline TRQ4 & 4,6 & Ausencia & Ausencia & Presencia & $\begin{array}{l}\text { Entoameba histolytica } \\
\text { Paramecio, Hifas de } \\
\text { Hongos }\end{array}$ \\
\hline GQ4 & 4,6 & Presencia & Ausencia & Presencia & Entoameba histolytica \\
\hline TRQ5 & 2,6 & Ausencia & Ausencia & Presencia & $\begin{array}{l}\text { Entoameba histolytica, } \\
\text { Paramecio, Hongos }\end{array}$ \\
\hline GQ5 & 4,6 & Ausencia & Ausencia & Presencia & Entoameba histolytica \\
\hline
\end{tabular}

Vertiente (V), tanque de captación (TC), tanque general (TG), grifo quesera (GQ), tanque reservorio (TR). Máximo permitido según la norma NTE INEN 1108: ${ }^{\star}<1,1 ;{ }^{* *}$ Ausencia

Tabla 1. Resultados del análisis microbiológico del sistema de agua que abastece a las queseras artesanales de la parroquia rural de Quimiag.

Los resultados del análisis físico-químico del agua que se presentan en la Tabla 2 indican que todas las muestras cumplen con los parámetros evaluados, lo que sugiere una homogeneidad entre los diferentes puntos de muestreo (8). Sin embargo, los datos sobre la carga microbiana sugieren la necesidad de la desinfección del agua tanto para uso doméstico como para las queseras.

Históricamente, la cloración es la tecnología más utilizada en la desinfección de agua; el cloro es un poderoso agente oxidante con acción germicida de amplio espectro (22), es económico, fácil de aplicar, con efecto residual y relativamente seguro en las dosis recomendadas $(23,24)$.

Pese a que los desinfectantes como el cloro/lejía pueden matar a la mayoría de los virus y bacterias que causan enfermedades, no son tan efectivos en el control de or- ganismos más resistentes, por lo que es necesario que el agua consumida en la comunidad deba ser tratada para eliminar o evitar la contaminación con estos parásitos.

Los quesos, aunque se caracterizan por ser seguros para el consumo, se han visto implicados en brotes transmitidos por los alimentos asociados con afecciones a la salud (26), por tanto, las deficiencias en los protocolos de limpieza y desinfección, sumados a la calidad del agua, son factores que no permitirán obtener un producto que garantice calidad e inocuidad, cumpliendo con los requisitos legales. 


\begin{tabular}{|c|c|c|c|c|c|c|c|c|}
\hline $\begin{array}{l}\text { PARÁME- } \\
\text { TRO }\end{array}$ & $\begin{array}{l}\text { TURBIEDAD } \\
\text { (U.N.T.) }\end{array}$ & $\begin{array}{c}\text { COLOR } \\
\text { (Und Pt-Co) }\end{array}$ & $\mathrm{pH}$ & $\begin{array}{c}\text { CONDUCTIVI- } \\
\text { DAD }(\mu \mathrm{S} / \mathrm{cm})\end{array}$ & $\begin{array}{l}\text { SÓLIDOS TO- } \\
\text { TALES (mg/L) }\end{array}$ & $\begin{array}{l}\text { NITRATOS } \\
(\mathrm{mg} / \mathrm{L})\end{array}$ & $\begin{array}{l}\text { NITRITOS } \\
(\mathrm{mg} / \mathrm{L})\end{array}$ & $\begin{array}{c}\text { FLORUROS } \\
(\mathrm{mg} / \mathrm{L})\end{array}$ \\
\hline $\begin{array}{l}{ }^{*} \text { MUESTRA/ } \\
{ }^{*} \text { MÁXIMO } \\
\text { PERMITIDO }\end{array}$ & 5 & 15 & $6,5-8,5$ & 1500 & 1000 & 50 & 3 & 1,5 \\
\hline V1 & $1,18 \pm 0,02$ & $0,00 \pm 0,00$ & $8,74 \pm 0,06$ & $280,33 \pm 1,52$ & $124,33 \pm 3,21$ & $1,33 \pm 0,06$ & $0,01 \pm 0,00$ & $0,18 \pm 0,02$ \\
\hline TC1 & $1,15 \pm 0,12$ & $0,00 \pm 0,00$ & $8,57 \pm 0,50$ & $193,67 \pm 5,13$ & $138,67 \pm 3,21$ & $1,57 \pm 0,12$ & $0,01 \pm 0,00$ & $0,58 \pm 0,04$ \\
\hline $\mathrm{V} 2$ & $3,31 \pm 0,34$ & $2,33 \pm 0,58$ & $8,30 \pm 0,36$ & $281,33 \pm 3,06$ & $93,33 \pm 5,03$ & $1,87 \pm 0,15$ & $0,01 \pm 0,00$ & $0,44 \pm 0,03$ \\
\hline TC2 & $0,77 \pm 0,07$ & $3,67 \pm 0,58$ & $8,37 \pm 0,32$ & $178,00 \pm 2,65$ & $107,0 \pm 2,00$ & $1,37 \pm 0,06$ & $0,017 \pm 0,006$ & $0,25 \pm 0,05$ \\
\hline V3 & $2,70 \pm 0,58$ & $3,60 \pm 2,08$ & $8,07 \pm 0,12$ & $110,67 \pm 1,15$ & $128,33 \pm 3,06$ & $2,33 \pm 0,06$ & $0,027 \pm 0,006$ & $0,59 \pm 0,006$ \\
\hline TC3 & $3,20 \pm 0,21$ & $13,53 \pm 0,50$ & $8,00 \pm 0,0$ & $117,33 \pm 2,52$ & $131,33 \pm 2,52$ & $2,37 \pm 0,06$ & $0,28 \pm 0,03$ & $0,60 \pm 0,00$ \\
\hline TR & $2,60 \pm 0,26$ & $9,67 \pm 1,53$ & $8,10 \pm 0,10$ & $156,67 \pm 2,08$ & $121,00 \pm 2,00$ & $1,57 \pm 0,12$ & $0,01 \pm 0,006$ & $0,57 \pm 0,03$ \\
\hline GQ1 & $1,10 \pm 0,08$ & $8,67 \pm 2,08$ & $7,23 \pm 0,21$ & $202,33 \pm 2,31$ & $122,67 \pm 2,08$ & $0,40 \pm 0,10$ & $0,10 \pm 0,006$ & $0,00 \pm 0,00$ \\
\hline GQ2 & $1,30 \pm 0,04$ & $1,32 \pm 0,04$ & $7,14 \pm 0,14$ & $195,33 \pm 1,53$ & $123,00 \pm 1,00$ & $0,67 \pm 0,12$ & $0,003 \pm 0,006$ & $0,00 \pm 0,00$ \\
\hline GQ3 & $2,40 \pm 1,37$ & $2,53 \pm 0,33$ & $7,04 \pm 0,40$ & $185,00 \pm 1,73$ & $122,54 \pm 2,00$ & $0,63 \pm 0,06$ & $0,01 \pm 0,01$ & $0,00 \pm 0,00$ \\
\hline CQ4 & $1,10 \pm 0,61$ & $1,13 \pm 0,66$ & $7,26 \pm 0,32$ & $180,00 \pm 1,00$ & $103,67 \pm 4,93$ & $1,47 \pm 0,06$ & $0,003 \pm 0,006$ & $0,00 \pm 0,00$ \\
\hline GQ4 & $1,10 \pm 0.66$ & $1,15 \pm 0,66$ & $7,41 \pm 0,02$ & $220,33 \pm 10,12$ & $105,67 \pm 1,53$ & $1,00 \pm 0,10$ & $0,01 \pm 0,00$ & $0,00 \pm 0,00$ \\
\hline CQ5 & $2,30 \pm 0,09$ & $2,26 \pm 0,09$ & $7,59 \pm 0,03$ & $222,67 \pm 1,53$ & $124,00 \pm 2,00$ & $1,17 \pm 0,15$ & $0,01 \pm 0,00$ & $0,004 \pm 0,004$ \\
\hline GQ5 & $1,60 \pm 0,15$ & $1,57 \pm 0,15$ & $7,15 \pm 0,05$ & $243,67 \pm 11,50$ & $118,33 \pm 3,06$ & $1,13 \pm 0,06$ & $0,003 \pm 0,006$ & $0,003 \pm 0,003$ \\
\hline
\end{tabular}

${ }^{*}$ Vertiente $(\mathrm{V})$, tanque de captación (TC), tanque general (TG), grifo quesera (GQ), tanque reservorio (TR). ${ }^{*}$ NTE INEN 1108 (Agua potable-requisitos)

Tabla 2. Resultados del análisis físico-químico del sistema de agua que abastece a las queseras artesanales de la parroquia rural de Quimiag.

\section{CONCLUSIONES}

El agua que abastece a la población de la parroquia Quimiag a pesar de cumplir $100 \%$ con los parámetros físico-químicos (color, conductividad, sólidos totales, turbiedad, nitratos, nitritos y fluoruros), sin embargo, no puede ser denominada potable ya que existen recuentos supe- riores a 1,1 respecto a coliformes totales y el $86 \%$ de las muestras presentan contaminación por Giardia lamblia.

Estos resultados inciden sobre la inocuidad de los quesos frescos artesanales elaborados en las queseras del sector, ya que se convierte en una fuente de contaminación microbiana. Se recomienda realizar un tratamiento previo con hipoclorito de sodio al 5 o $10 \%$ antes de consumirla y utilizarla en la producción de quesos.

1. Arispe I, Tapia M. Inocuidad y calidad: requisitos indispensables para la protección de la salud de los consumidores. Agroalimentaria. 2017; 13: 105-117.

2. Fente-Sampayo C, Vazquez-Belda B, Franco-Abuin C, Quinto-Fernandez E, Cepeda-Saez A. Influencia de las buenas prácticas de fabricación en la contaminacion fúngica del queso de Arzúa. Cienc. Tecnol. Aliment. 2001; 3: 169-172.

3. Godi K. The Microbiological quality of raw milk after introducing the two day â€TM s milk collecting system. 2008; 1: 61-74.

4. De Silva S, Kanugala K, Weerakkody N. Microbiological quality of raw milk and effect on quality by implementing good management practices. Procedia Food Science. 2016;6: 92-6.

5. Arguello P, Lucero O, Castillo G, Escobar S, Albuja A, et al. Calidad microbiológica de los quesos artesanales elaborados en zonas rurales de Riobamba (Ecuador). Perspectiva. 2015; 16: 65-74.

6. Arguello P, Escobar S, Espinoza C, Albuja A. Calidad microbiológica de la materia prima, suero y producto terminado en las queseras artesanales: Quimiag-Ecuador. En: Sin editor. Libro de Memorias. VII Congreso iberoamericano de Ciencias Farmacéuticas (COIFFA); 29 y 30 noviembre, 1 
diciembre 2017, Riobamba. Ecuador: Sin editor; 2018. P. 156-162.

7. Nerín C, Aznar M, Carrizo D. Food contamination during food process. Trends in Food Science \& Technology. 2016; 48: 63-68.

8. NORMA NTE. INEN 2169:2013. Calidad del agua. Muestreo. Manejo y conservación de muestras.

9. NORMA NTE. INEN 1105:2012 Aguas. Muestreo para exámen microbiológico.

10. NORMA MEXICANA NMX-AA-042-SCFI-2015. Análisis de agua - enumeración de organismos coliformes totales, organismos coliformes fecales (termotolerantes) y Escherichia coli - método del número más probable en tubos múltiples.

11. Arnedo I, Bracho M, Díaz-Suárez O, Botero L. Técnicas para la detección de Cryptosporidium sp. en sistemas de tratamiento de agua residual. Kasmera [Internet]. 2008 [citado 1 Enero 2018]; 3: 120-128. Disponible en: http://www.scielo.org.ve/scielo.php?script=sci_arttext\&pi$\mathrm{d}=$ S0075-52222008000200004\&lng=es.

12. Pérez-Cordón Gregorio, Rosales María J., Valdez Renzo A., Vargas-Vásquez Franklin, Cordova Ofelia. Detección de parásitos intestinales en agua y alimentos de Trujillo, Perú. Rev. Perú. Med. Exp. Salud Pública [Internet]. 2008 [citado 15 Mayo 2018]; 25: 144-148. Disponible en: http://www. scielo.org.pe/scielo.php?script=sci_arttext\&pid=S1726-46342008000100018\&lng=es.

13. NORMA NTE. INEN 1108:2014. Agua potable requisitos.

14. Abu R. Water Chemestry and Microbiology. En: Chormey D, Bakirdere S, Turan N, Engin G, editores. Comprehensive Analytical Chemistry, Vol 81.

Netherlands: Elsevier; 2018. p. 1-56.

15. Silva E, Murillo C, Nava G, Cárdenas O, Peralta A, et al. Microbiological and chemical quality of water used in Colombian food industries. Biomédica. 2010; 30: 421-431.

16. García RR, Muñoz CM, Vizcaino DH, Veguillas DL, Acevedo L. Calidad del agua de fuentes de manantial en la zona básica de salud de Sigüenza. Rev Esp Salud Pública [Internet]. 2003 [citado 30 Julio 2018]; 77: 423-32. Disponible en: https://www.redalyc.org/pdf/170/17077312.pdf

17. Rodríguez S, Asmundis C, Ayala M, Arzú, O. Presencia de indicadores microbiológicos en agua para consumo humano en San Cosme (Corrientes, Argentina). Revista veterinaria [Internet]. 2018 [citado 25 Junio 2018]; 29: 9-12. Disponible en: http://revistas.unne.edu.ar/index.php/vet/article/ view/2779/2460

18. Arcos D, Ávila S, Estupiñán M, Gómez A. Indicadores microbiológicos de contaminación de las fuentes de agua. Nova [Internet]. 2005 [consultado 25 Julio 2018]; 3: 69-79. Disponible en: http:// www.unicolmayor.edu.co/invest_nova/NOVA/ARTREVIS2_4.pdf

19. Daivila J, Reyes G, Corzo O. Evaluación microbiológica de las diferentes etapas del proceso de elaboración de queso tipo Gouda en una industria venezolana. Arch Latinoam Nutr [Internet]. 2006 [consultado 30 Julio 2018]; 56: 1-10. Disponible en: https://www.researchgate.net/publication/6996893_Microbiological_assessment_of_the_Gouda-type_cheese-making_process_in_a_ Venezuelan_industry

20. Caccio S, Chalmers R. Human cryptosporidiosis in Europe. Clin. Microbiol. Infect. 2016; 22: 471-480.

21. Ryan U, Cacciò S. Zoonotic potential of Giardia. International journal for parasitology. 2013; 43: 943-956.

22. Cerqueira M, Picinin L, Fonseca L, Souza M, Leite M, et al. Microbiological quality of milk through preventive techniques in the handling of production. Ciênc. agrotec. [Internet]. 2005 [consultado 30 Julio 2018]; 29: 216-222. Disponible en: http://www.scielo.br/pdf/cagro/v29n1/a27.pdf

23. Clasen T, Schmidt W, Rabie T, Roberts I, Cairncross S. Interventions to improve water quality for preventing diarrhoea: systematic review and meta-analysis. BMJ. 2007; 334: 782-782.

24. Burke N, Dwyer J, Southern M, Adley C. An analysis of the microbial quality of water in a milk production plant. Food Sci Technol [Internet]. 2017 [citado 15 Septiembre 2018]; 78: 41-46. Disponible en: https://www.sciencedirect.com/science/article/pii/S0023643816307277 
25. Morán M, Ochoa T. Prevención, diagnóstico y tratamiento de infecciones pediátricas en desastres naturales. Rev Peru Med Exp Salud Publica [Internet]. 2017 [citado 15 Octubre 2018]; 34: 723 730. Diposnible en: https://www.scielosp.org/scielo.php?pid=S172646342017000400723\&script=sci_arttext\&tlng=en

26. Kousta M, Mataragas M, Skandamis P, Drosinos E. Prevalence and sources of cheese contamination with pathogens at farm and processing levels. Food Control. 2010; 21: 805-815. 